\title{
BILATERAL GENERATING FUNCTIONS FOR A NEW CLASS OF GENERALIZED LEGENDRE POLYNOMIALS
}

A.N. SRIVASTAVA, S.D. SINGH and S.N. SINGH

Department of Mathematics

Banaras Hindu University

Varanasi-221005, India

(Received June 16, 1978 and in Revised form August 29, 1978)

ABSTRACT. Recently Chatterjea (1) has proved a theorem to deduce abilateral generating function for the Ultraspherical polynomials. In the present paper an attempt has been made to give a general version of Chatterjea's theorem. Finallys the theorem has been specialized to obtain a bilateral generating function for a class of polynomials $\left\{\mathrm{P}_{\mathrm{n}}(\mathrm{x} ; \alpha, \beta)\right\}$ introduced by Bhattacharjya (2). KEY WORDS AND PHRASES. Bilateral generating function, Ultraspherical polynomials, Legendre polynomials, Orthogonal polynomials, Weight function, Rodrigue's formula.

1980 MATHEMATICS SUBJECT CLASSIFICATION CODES. $42 A 52$.

Primary 33A45, 33A65; Secondary 


\section{INTRODUCTION.}

Using the following differential formula for the Ultraspherical polynomials $\mathrm{P}_{\mathrm{n}}^{\lambda}(\mathrm{x})$ due to Tricomi,

$$
P_{n}^{\lambda}\left[x\left(x^{2}-1\right)^{-1 / 2}\right]=\frac{(-1)^{n}}{n} !^{-}\left(x^{2}-1\right)^{\lambda+\frac{n}{2}} D^{n}\left(x^{2}-1\right)^{-\lambda},
$$

Chatterjea (1) has recently obtained a bilateral generating function for the U1traspherical polynomials in the form of following theorem.

THEORRM 1. If

$$
F(x, t)=\sum_{m=0}^{\infty} a^{m} t^{m} P_{m}^{\lambda} \quad(x),
$$

then

$$
\rho^{-2 \lambda} F\left(\frac{x-t}{\rho}, \frac{t y}{\rho}\right)=\sum_{r=0}^{\infty} t^{r} \quad b_{r}(y) P_{r}^{\lambda}(x),
$$

where

$$
b_{r}(y)=\sum_{m=0}^{\infty}\left(\begin{array}{l}
r \\
m
\end{array}\right) a_{m} y^{m} \text {, and } \rho=\left(1-2 x t+t^{2}\right)^{1 / 2} \text {. }
$$

A closer look at the above relation (1.2) suggests the following interesting general version of Chatterjea's theorem:

2. Let $F \circ G$ be used to denote the composition $F \circ G(x)=F(G(x))$. In terms of this notation, we state

THEOREM 2. Suppose that there exist functions $f, g, h$ and $X$ and $a$ sequence of constants $\left\{c_{n}\right\}$ such that the sequence of functions $\left\{Q_{n}\right\}$ is generated by the formula 


$$
c_{n} f g^{n} Q_{n} \circ x=D^{n} h, \quad n=0,1,2, \ldots,
$$

where $D \equiv d / d x$. Define the generating function

$$
F(x, t)=\sum_{n=0}^{\infty} a_{n} t^{n} Q_{n}(x) .
$$

Then

$$
\left.f F(X, g t z)\right|_{x+t}=f \sum_{n=0}^{\infty} c_{n}(g t)^{n} Q_{n} 0 x b_{n}(z),
$$

where

PROOF. By Taylor's theorem,

$$
b_{n}(z)=\sum_{k=0}^{\infty} \frac{a_{k}}{c_{k}(n-k) !} z^{k} .
$$

$$
\left.f F(x, g t z)\right|_{x+t}=e^{t D} f F(x, g t z) .
$$

To evaluate the right hand side of (2.3), we shall use as our starting point the relations (2.1) and (2.2), and the series expansion for $e^{t D}$. Thus

$$
\begin{aligned}
e^{t D} f F(x, g t z) & =e^{t D} f \sum_{n=0}^{\infty} a_{n}(g t z)^{n} Q_{n} \circ x \\
& =e^{t D} \sum_{n=0}^{\infty} \frac{a_{n}}{c_{n}}(t z)^{n} D^{n} h \\
& =\sum_{n=0}^{\infty} \sum_{m=0}^{\infty}\left(a_{n} / c_{n}\right) t^{n+m} z^{n} D^{n+m} h / m ! \\
& =\sum_{n=0}^{\infty} \sum_{m=0}^{\infty}\left(a_{n} / c_{n}\right)(g t)^{n+m} c_{n+m} f Q_{n+m} \circ x / m ! \\
& =f \sum_{n=0}^{\infty} c_{n}(g t)^{n} Q_{n} \circ x b_{n}(z),
\end{aligned}
$$

where

$$
b_{n} \cdot(z)=\sum_{k=0}^{\infty} \frac{a_{k}}{c_{k}(n-k) !} z^{k} \text {. }
$$

It is worthwhile to remark here that if we choose $Q_{n}(x)=P_{n}^{\lambda}(x)$, $f(x)=\left(x^{2}-1\right)^{-\lambda}, g(x)=\left(x^{2}-1\right)^{-1 / 2}, x(x)=x\left(x^{2}-1\right)^{-1 / 2}, h(x)=\left(x^{2}-1\right)^{-\lambda}$ and $c_{n}=n ! /(-1)^{n}$ then Theorem? would correspond to Chatterjea's theorem. APPLICATIONS: Earlier, Bhattacharjya (2) introduced a new class of generalized Legendre polynomials $\left\{\mathrm{P}_{n}(\mathrm{x} ; \alpha, \beta)\right\}$ which are orthogonal with the 


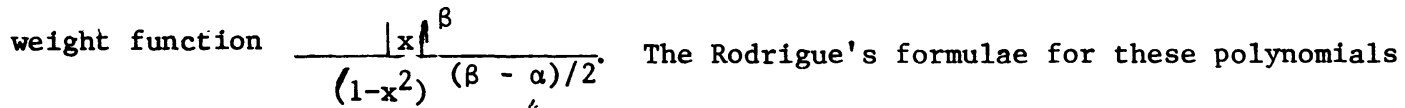
are $(2,(6.6)$ and $(6.8))$ :

$$
\begin{aligned}
P_{2 m}\left(x^{-1 / 2} ; \alpha, \beta\right)= & \frac{x^{m+(\alpha+1) / 2}(1-x)(\beta-\alpha) / 2}{(-2 m-(\alpha-1) / 2)_{m}} \\
& \left.\cdot D^{m[}(1-x)^{m-(\beta-\alpha) / 2} x^{-m-(\alpha+1) / 2}\right],
\end{aligned}
$$

and

$$
\begin{aligned}
P_{2 m+1}\left(x^{-1 / 2} ; \alpha, \beta\right)= & \frac{x^{m+1+\alpha / 2}(1-x)(\beta-\alpha) / 2}{(-2 m-(\alpha+1) / 2)_{m}} \\
& \cdot D^{m\left[(1-x)^{m-(\beta-\alpha) / 2} x^{-m-(\alpha+3) / 2}\right]}
\end{aligned}
$$

Here we note that the sequences $\left\{P_{2 n}\left(x^{-1 / 2} ; \alpha-2 n, \beta\right)\right\}$ and $\left\{P \frac{2 n+1}{\left(x^{-1 / 2}\right.}\right.$; $\alpha-2 n, \beta)\}$ are amenable to a method of Theorem 2 for finding bilateral generating ifunctions.

Let $Q_{n}(x)=P_{2 n}(x ; \alpha-2 n, \beta) \equiv P_{2 n}(x)$. For simplicity of notation, set $y=-(\alpha+1) / 2$ and $\delta=(\alpha-\beta) / 2$. Then $(2.1)$ holds with $f(x)=x^{y}(1-x)^{\delta}$, $g(x)=(1-x)^{-1}, \quad x(x)=x^{-1 / 2}$ and $c_{n}=\phi(n)=(-n-(\alpha-1) / 2)_{n}$. Upon replacing $t$ by $-t$ and $z$ by $-y$, we get

$$
\begin{array}{r}
\left(\frac{x-t}{x}\right)^{y}\left(\frac{1-(x-t)^{\delta}}{1-x}\right) F\left(\frac{1}{(x-t)^{1 / 2}},-\frac{y t}{(1-(x-t))}\right)=\sum_{r=0}^{\infty}\left(\frac{-t}{1-x}\right)^{r} \phi(r) \cdot \\
. P_{2 r}\left(x^{-1 / 2}\right) b_{r}(-y),(2.6)
\end{array}
$$

where

and

$$
F\left(\frac{1}{x^{1 / 2}}, \frac{t}{1-x}\right)=\sum_{m=0}^{\infty} a_{m}\left(\frac{t}{1-x}\right)^{m} P_{2 m}\left(x^{-1 / 2}\right)
$$

$$
b_{r}(-y)=\sum_{m=0}^{\infty} \frac{a_{m}(-y)^{m}}{\phi(m)(r-m) !} .
$$

Now replacing $x^{-1 / 2}$ by $s$ and $t /(1-x)$ by $t$ in $(2.6)$, we are led to the following

bilateral generating function for gerneralized even Legendre polynomials:

COROLLARY. 1 : If

$$
F(x, t)=\sum_{m=0}^{\infty} a_{m} t^{m} P_{2 m}(x)
$$


then

$$
\left[1-\left(x^{2}-1\right) t\right]^{y} \quad(1+t)^{\delta} F\left(\frac{x}{\left(1-t\left(x^{2}-1\right)\right)^{1 / 2}}, \frac{y t}{1+t}\right)=\sum_{r=0}^{\infty}(-t)^{r} \phi(r) .
$$

where $b_{r}(-y)$ is given by (2.7).

$$
\text { - } P_{2 r}(x) b_{r}(-y) \text {, }
$$

In the same way, let $Q_{n}(x)=P_{2 n+1}(x ; \alpha-2 n, \beta) \equiv P_{2 n+1}(x)$, and set $y=-(\alpha+2) / 2, \delta=(\alpha-\beta) / 2$. Then $(2.1)$ holds with $f(x)=x^{y}(1-x)^{\delta}$, $g(x)=(1-x)^{-1}, x(x)=x^{-1 / 2}$ and $c_{n}=\psi(n)=(-n-(\alpha+1) / 2)_{n}$. Replacing $t$ by $-t$ and. $z$ by $-y$ and making the same substitution as before in (2.7), we are led to the following bilateral generating function for generalized odd Legendre polynomials. CGROLLARY 2: If

$$
F(x, t)=\sum_{m=0}^{\infty} a_{m} t^{m} P_{2 m+1}(x),
$$

then

$$
\begin{aligned}
{\left[1-\left(x^{2}-1\right) t\right]^{y}(1-t)^{\delta} F\left(\frac{x}{\left(1-t\left(x^{2}-1\right)\right)^{1 / 2}}, \frac{t y}{1+t}\right)=} & \sum_{r=0}^{\infty}(-t)^{r} \psi(r) \cdot \\
& \cdot P_{2 r+1}(x) c_{r}(-y),
\end{aligned}
$$

where

$$
c_{r}(-y)=\sum_{m=0}^{F} \frac{a_{m}(-y)^{m}}{\Psi(m)(r-m) !} .
$$

Taking $\alpha=\beta$ in Corollary 1 and 2 , we can obtain bilateral generating functions for generalized Legendre polynomials due to Dutta and More (3).

Next, we note that (2),

$$
\begin{aligned}
& \qquad P_{2 m}(x ; 0,0)=\frac{(-1)^{m} m ! P_{2 m}(x)}{\left(-2 m+\frac{1}{2}\right)_{m}}, \\
& \text { and } \\
& \qquad P_{2 m}(x ; 0,0)=\frac{(-1)^{m} m ! P_{2 m+1}(x)}{\left(-2 m-\frac{1}{2}\right)_{m}},
\end{aligned}
$$

where $P_{2 m}(x)$ and $P_{2 m+1}(x)$ are even and odd Legendre polynomials. Therefore, by (2.8), (2.9) and the above two corollories we can obtain bilateral generating functions for Legendre polynomials. 
Acknowledgements :

The authors wish to express their thanks to the referee for his valuable suggestions for the improvement of this paper.

REFERENCES

1. CHATTERJEA, S.K.,A Bilateral generating Function for the U1traspherical Polynomials, Pacific J. Math. 29 (1969) 73-76.

2. BHATTACHARJYA, M., On Some Generalisation of Legendre Polynomials, Bull. Cal. Math. Soc. 66 (1974) 77-85.

3. DUTTA, M. and MORE, K.L., A New Class of Generalised Legendre Polynomials, Mathematica (Cluj) 7(30) (1965) 33-41. 


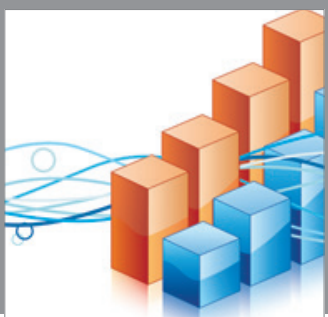

Advances in

Operations Research

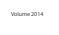

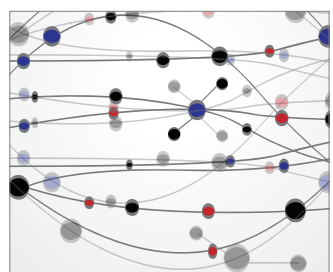

\section{The Scientific} World Journal
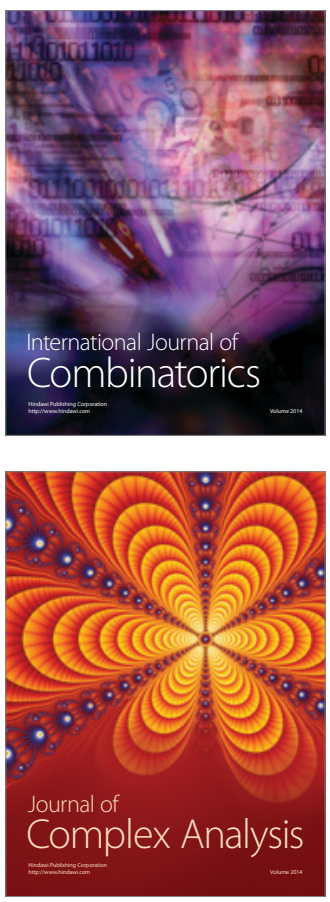

International Journal of

Mathematics and

Mathematical

Sciences
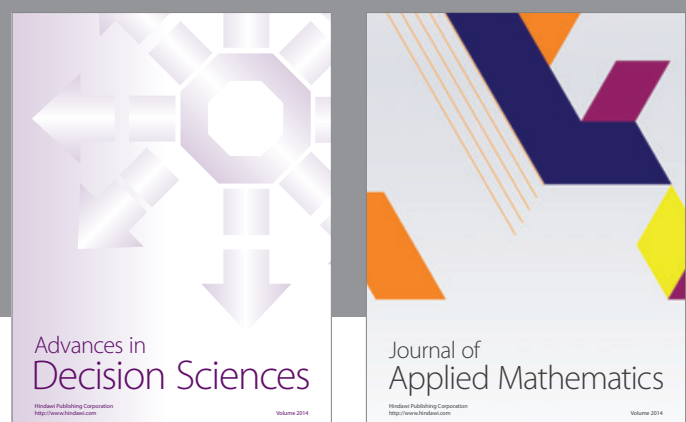

Journal of

Applied Mathematics
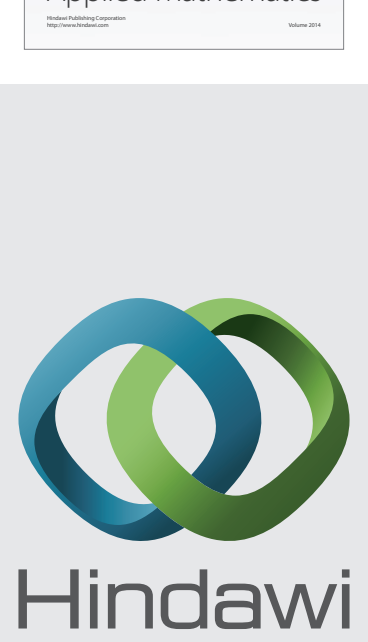

Submit your manuscripts at http://www.hindawi.com
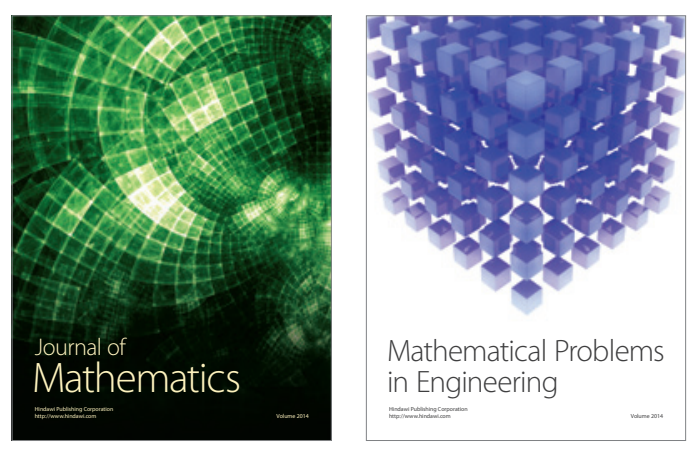

Mathematical Problems in Engineering
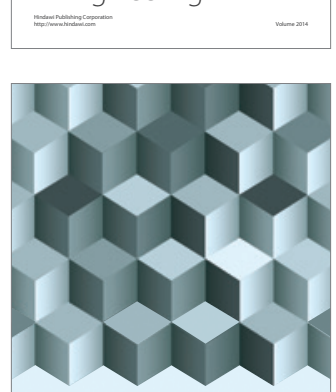

Journal of

Function Spaces
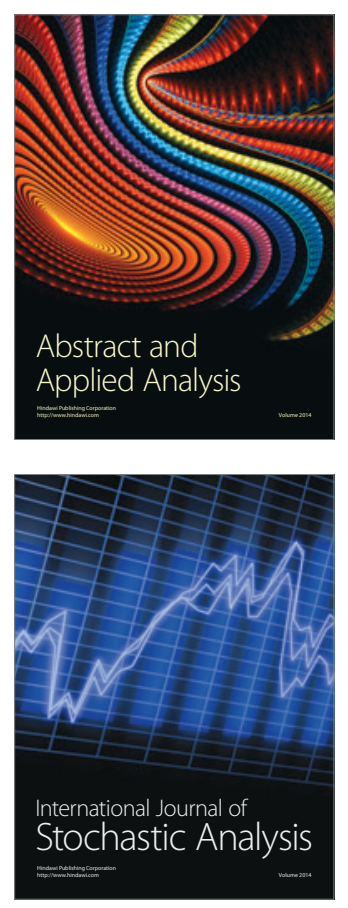

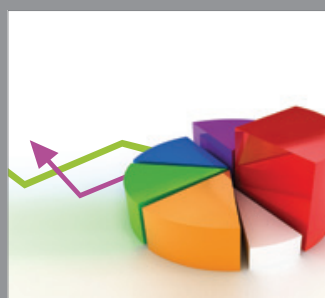

ournal of

Probability and Statistics

Promensencen
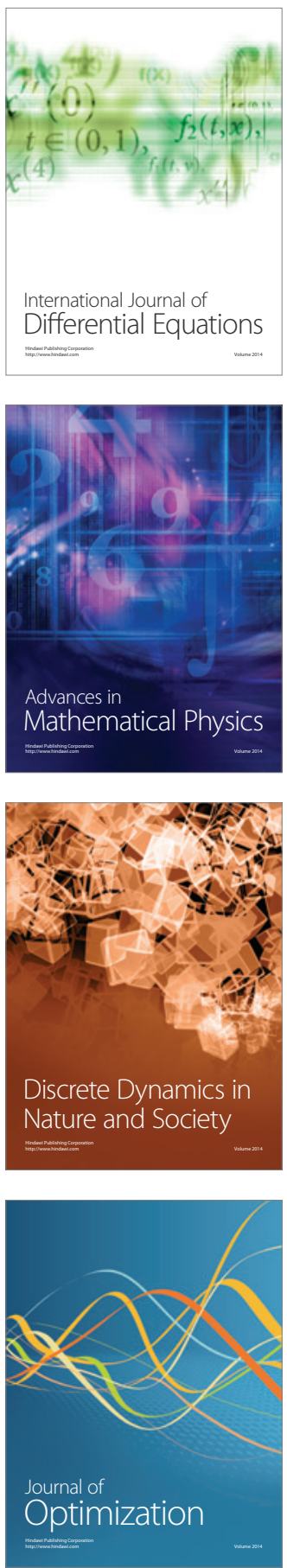